This item was submitted to Loughborough's Institutional Repository (https://dspace.lboro.ac.uk/) by the author and is made available under the following Creative Commons Licence conditions.

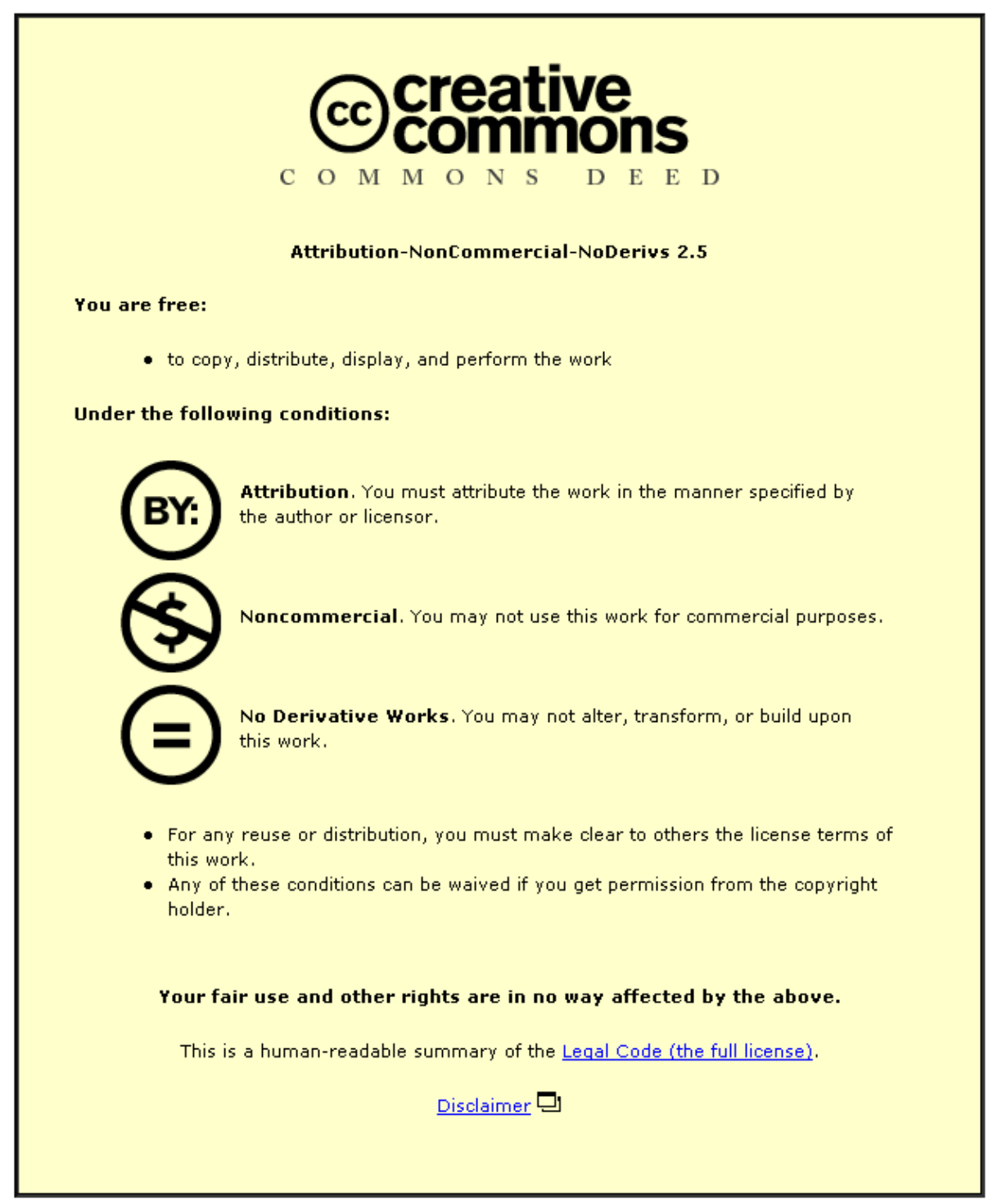

For the full text of this licence, please go to: http://creativecommons.org/licenses/by-nc-nd/2.5/ 


\title{
Nonlinear Multilevel Dynamics of a Coupled SQUID Ring-Resonator System in the Hysteretic Regime
}

\author{
R. J. Prance, R. Whiteman, T. D. Clark,* H. Prance, V. Schöllmann, J. F. Ralph, S. Al-Khawaja, and M. Everitt \\ School of Engineering, University of Sussex, Brighton, Sussex BN1 9QT, United Kingdom
}

(Received 16 October 1998)

\begin{abstract}
We consider the dynamical behavior of a strongly hysteretic SQUID ring coupled to a radio frequency resonator. By experiment we show that this system can display novel multiple level structures in its rf voltage-current characteristics which are solutions of the nonlinear equations of motion describing the system. [S0031-9007(99)09459-4]

PACS numbers: $85.25 . \mathrm{Dq}, 74.50 .+\mathrm{r}$
\end{abstract}

The usefulness of the SQUID magnetometer [here, a weak link enclosed by a thick superconducting ring inductively coupled to a parallel $L C$ resonant (tank) circuitFig. 1(b)] is well known [1]. Its sensitivity relies on the $\Phi_{0}(=h / 2 e)$ periodic response to an applied magnetic flux $\Phi_{x}$. This response is manifestly nonlinear and can give rise to chaotic behavior [1-4] which can adversely affect the performance of SQUIDs as magnetometers. However, the nonlinear dynamics of SQUID circuit systems may also generate other very interesting and potentially useful behavior. An example is stochastic resonance [5] which can occur where multiple stable states coexist. In this paper we show that a new class of multiply coexisting dynamical states can develop in a driven SQUID ringradio frequency (rf) tank circuit system when the ring is operating in the strongly hysteretic regime.

SQUID ring regimes of behavior can be categorized in terms of the parameter $\beta=2 \pi \Lambda I_{\text {crit }} / \Phi_{0}$, where $I_{\text {crit }}$ is the weak link critical current and $\Lambda$ is the ring inductance. Thus, the inductive $(\beta \leq 1)$ and hysteretic $(\beta>1)$ regimes correspond to the ring screening current $I_{s}$, or total included magnetic flux $\Phi$, being either single or multivalued in $\Phi_{x}^{1}$. In the hysteretic regime, $I_{s}\left(\Phi_{x}\right)$ consists of a set of approximately diamagnetic branches with extremal values on any branch set by $I_{\text {crit }}$. In the absence of noise, jumps between adjacent branches occur at $\pm I_{\text {crit }}$ so that for excursions $\Phi_{x}>\Lambda I_{\text {crit }}$ hysteresis loops are traced out in $I_{s}\left(\Phi_{x}\right)$. These loops form the basis of the hysteretic SQUID magnetometer [1,6] where the (time dependent) flux $\Phi_{x}(t)$ is supplied via a coupled tank circuit, typically resonant at rf. Ordinarily, it is assumed that the ring stays at, or extremely close to, the minimum in its potential [1]

$$
U\left(\Phi, \Phi_{x}\right)=\frac{\left(\Phi-\Phi_{x}\right)^{2}}{2 \Lambda}-\frac{I_{\text {crit }} \Phi_{0}}{2 \pi} \cos \left(\frac{2 \pi \Phi}{\Phi_{0}}\right),
$$

so following $\Phi_{x}(t)$ adiabatically. In this situation the ring-tank circuit dynamics ( $\mathrm{rf}$ tank circuit voltage $V_{\text {out }}$ vs $r f$ input current $I_{\text {in }}$ ) are of the standard hysteretic SQUID magnetometer form, i.e., a set of steplike features in $V_{\text {out }}$ vs $I_{\text {in }}$, periodic in $I_{\text {in }}$, with a current interval set by $\Phi_{0} / \Lambda$. In general, as successive pairs of hysteresis loops in $I_{s}\left(\Phi_{x}\right)$ are swept out with increasing $\Phi_{x}(\mathrm{rf})$, a new step is generated in $V_{\text {out }}$ vs $I_{\text {in }}$. Each step is modulated (modulo $\Phi_{0}$ ) by a static or quasistatic applied flux $\left(\Phi_{\text {xqstat }}\right)$. We will refer to the $V_{\text {out }}$ vs $I_{\text {in }}$ for $\Phi_{\text {xqstat }}=n \Phi_{0}$ or $(n+1 / 2) \Phi_{0}, n$ integer, as the extremal characteristics. In the standard treatment a comparison of the rf voltages at which the first step starts on these extremal characteristics (the breakpoints) yields [6] $I_{\text {crit }}$ and hence $\beta$. It was recognized early on [7] that the adiabatic constraint on ring-tank circuit dynamics can break down, at least when the circuit parameters lead to underdamping. With this constraint removed, the ring need not necessarily follow the minimum in $U\left(\Phi, \Phi_{x}\right)$ as $\Phi_{x}(t)$ varies, implying that the simple hysteresis loop model may no longer provide an accurate picture for $I_{s}(t)$. As we shall see, the lifting of the constraint at large $\beta$ values leads to quite different dynamical characteristics to those usually observed.

As we have recently demonstrated [8], improved rf receivers have helped us uncover new inductive mode ring-tank circuit dynamics. We have now used these receivers to probe the hysteretic regime. In particular, we have concentrated on exploring the phase space for which $\beta$ is large (i.e., $\beta \approx 30-50$ ). The $\mathrm{rf}$ electronics, used to make rf diode detected plots of $V_{\text {out }}$ vs $I_{\text {in }}$, consisted of a low noise, $4.2 \mathrm{~K} \mathrm{GaAsFET}$ preamplifier (gain close to $20 \mathrm{~dB}$ ) and a low noise, room temperature receiver [8] [see Fig. 1(b)]. The SQUID rings studied were of the Zimmerman two hole type [9], fabricated in niobium, in which the enclosed weak link was a niobium point contact, allowing us to access a wide range of $\beta$ values.

In general, large $\beta$ dynamical characteristics do not display the familiar (adiabatic) steps in $V_{\text {out }}$ vs $I_{\text {in }}$. Instead, gross step structures develop on $V_{\text {out }}$ and $I_{\text {in }}$ scales large compared with those observed using standard SQUID magnetometers $[1,6]$. Furthermore, for certain ranges of ring parameters, these large $\beta$ characteristics contain novel, and reproducible, multilevel structures. An experimental, diode detected example is shown in Fig. 1(a) (taken at $4.2 \mathrm{~K}$ with $\Phi_{\text {xqstat }}=n \Phi_{0}$, a measured system quality factor $Q=500$, and a coupling constant squared $K^{2}=M^{2} / L_{t} \Lambda=0.008$ for tank circuit inductance $L_{t}$ 
and ring-tank circuit mutual inductance $M$ ). As is common practice, this characteristic was recorded at the $n \Phi_{0}$ bias frequency $f_{\text {rf }}$ (here $23.02 \mathrm{MHz}$ ) equal to the resonant frequency for the coupled system when $\Phi_{\mathrm{xqstat}}=n \Phi_{0}$. As can be seen, the gross structure in Fig. 1(a) contains four distinct sublevels. However, as we shall see, this number is dependent on the circuit parameters of the coupled system. At larger amplitudes of $I_{\text {in }}$ (not shown in Fig. 1) the gross structure is repeated periodically in $I_{\text {in }}$ although after the first structure those which follow may
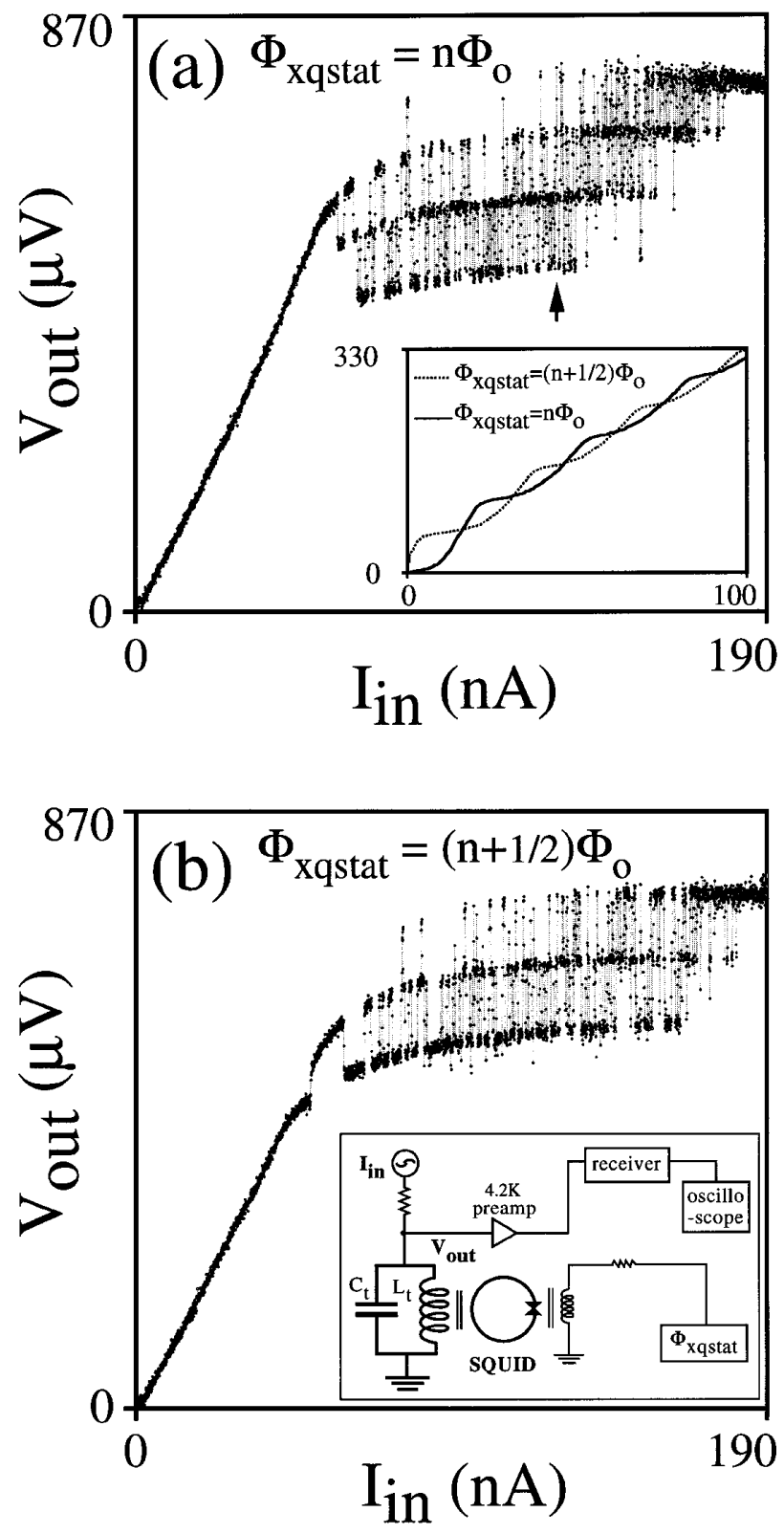

FIG. 1. Large $\beta$, diode detected, $4.2 \mathrm{~K}$ dynamics; $\left(f_{\mathrm{rf}}=\right.$ $\left.23.02 \mathrm{MHz}, Q=500, K^{2}=0.008\right)$ at $\Phi_{\text {xqstat }}=n \Phi_{0}$ and $(n+1 / 2) \Phi_{0}, n$ integer. Insets in (a) $4.2 \mathrm{~K}$ adiabatic dynamics at $\Phi_{\text {xqstat }}=n \Phi_{0}$ and $(n+1 / 2) \Phi_{0}$ with $\beta$ just less than one $\left(f_{\mathrm{rf}}=23.02 \mathrm{MHz}, Q=500, K^{2}=0.008\right)$ and in (b) system block diagram. be very smeared out. With sufficient resolution these periodically repeated gross structures are always found to contain the same number of sublevels, albeit less distinct than those plotted in Fig. 1(a). When $\Phi_{\text {xqstat }}$ is changed from $n \Phi_{0}$ to $(n+1 / 2) \Phi_{0}$ the sublevels move in $V_{\text {out }}$ and the number is reduced by one [here, from 4 to 3 - see Fig. 1(b)]. This was the case for all the large $\beta$, sublevel structure characteristics we studied. We note that from an adiabatic viewpoint the characteristics in Figs. 1(a) and 1(b) would appear to display multiple critical currents, one for each matching pair of extremal breakpoints. However, as we shall see, these sublevel structures can be modeled on the basis of just one critical current $\left(I_{\text {crit }}\right)$. We also note that any estimate of $\beta$ involving gross structure characteristics must rely on a best fit choice of $I_{\text {crit }}$ to calculate the system dynamics.

For comparison with the Fig. 1 dynamics, we show inset standard (adiabatic) $4.2 \mathrm{~K}$ SQUID magnetometer characteristics taken using the ring-tank circuit system of Fig. 1. Here, with all other circuit parameters held the same, the weak link was readjusted to make $\beta$ very close to one. Clearly, compared with these characteristics the large $\beta$ dynamics display sublevels which are very extended along the $I_{\text {in }}$ axis. Each of these sublevels has the same separation in $V_{\text {out }}$ as the standard characteristics, implying a system response $\Phi_{0}$ periodic in $\Phi_{\text {xqstat }}$, as is observed experimentally. We also note that the length of the sublevels in the gross step structures is always an integer multiple of the step length seen on the adiabatic characteristics. We have recorded a whole range of these gross structures with varying numbers of sublevels, from a minimum of 2 (at $\Phi_{\text {xqstat }}=n \Phi_{0}, T=4.2 \mathrm{~K}$ ) to a maximum of $13\left(\Phi_{\text {xqstat }}=n \Phi_{0}, T=1.9 \mathrm{~K}\right)$. To add to Fig. 1 we show in Fig. 2 another example of these structures, taken for a different ring-tank circuit system where $Q=500$, $K^{2}=0.004, f_{\mathrm{rf}}=20.019 \mathrm{MHz}$, and $T=4.2 \mathrm{~K}$. Here, with the two extremal characteristics overlapped $\left[n \Phi_{0}-\right.$ filled circles, $(n+1 / 2) \Phi_{0}$ - open circles], the first two gross structures can be seen, both displaying three $\left(n \Phi_{0}\right)$ or two $\left[(n+1 / 2) \Phi_{0}\right]$ sublevels.

It is clear in Fig. 1 that stochastic jumps can occur between sublevels. These jumps (with submillisecond dwell times in any particular sublevel) presumably arise because of the classical noise in the system. Since the ring-tank circuit coupling is weak, with the latter driven by a $4.2 \mathrm{~K}$ source, it is reasonable to assume that this noise comes from the tank circuit itself. The jumps can be better appreciated by examining the time domain record of the sublevels in Fig. 1, as shown in Fig. 3(a). Here, with the peak $I_{\text {in }}$ set as arrowed in Fig. 1(a), and $\Phi_{\text {xqstat }}=n \Phi_{0}$, we see apparently random time jumping between a set of equally spaced levels. The phase space portrait of these processes is revealing. In Fig. 3(b) we show this (pseudo) phase portrait for the continuing data set of Fig. 3(a). As is customary, we plot $V_{\text {out }}(t)$ against the time shifted 


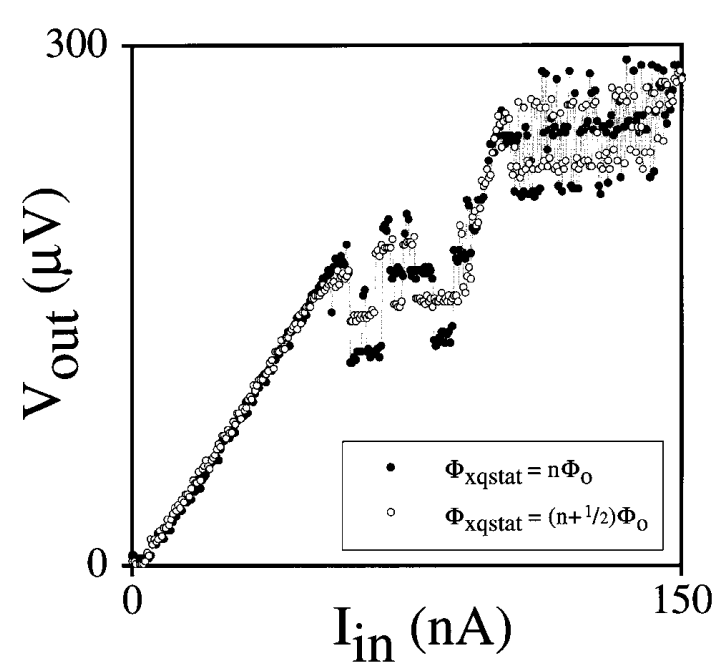

FIG. 2. Large $\beta$, diode detected $4.2 \mathrm{~K}$ characteristics showing three and two sublevels, respectively, at $\Phi_{\mathrm{xqstat}}=n \Phi_{0}$ and $(n+1 / 2) \Phi_{0}\left(f_{\mathrm{rf}}=20.019 \mathrm{MHz}, Q=500, K^{2}=0.004\right)$.

$V_{\text {out }}(t+\tau)$ at all times $t$, where $\tau$ is a suitable time delay $\left(10^{-4} \mathrm{sec}\right.$ in this example). We note that the number of return points (nodes), where the ellipses intercept on the phase portrait, is equal to the number of levels, as it should be. We also note that if $\tau \rightarrow 0$ the ellipses in
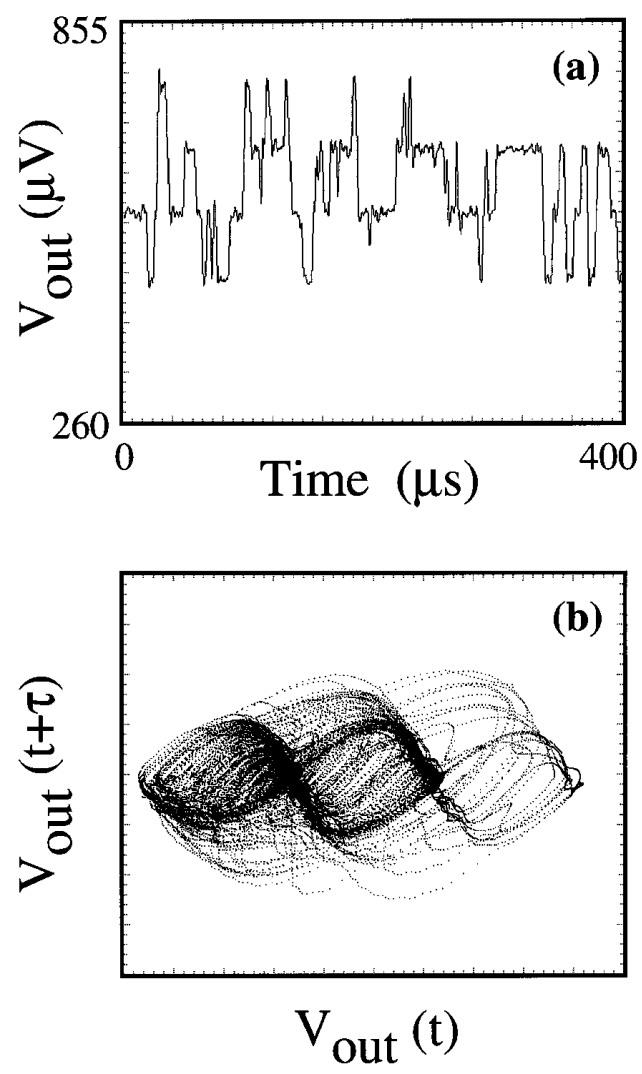

FIG. 3. (a) Diode detected, time domain plot of the level voltages [at the peak $I_{\text {in }}$ arrowed in Fig. 1(a)] showing transitions (b) $V(t)$ vs $V(t+\tau)$ for the time domain voltage levels of (a) where $\tau=10^{-4}$ sec.
Fig. 3(b) close down to a point, while if $\tau \rightarrow \infty$ these ellipses become completely washed out.

In modeling the structure typified by Fig. 1, we have used the resistively shunted junction plus capacitance $(\mathrm{RSJ}+C)$ description of a weak link [1,2]. Given that the $Q$ is large $(\approx 500)$, the resonant frequency of the bare tank circuit (capacitance $C_{t}$, parallel resonance resistance $\left.R_{t}\right)$ is given by $f_{R}=\omega_{R} / 2 \pi=1 / 2 \pi \sqrt{L_{t} C_{t}}$, with $R_{t}=$ $Q \omega_{R} L_{T}$. The magnetic fluxes, $\Phi$ in the ring (ring current $I_{S}$ ) and $\varphi$ in the tank circuit inductor (inductor current $I_{L_{t}}$, are then $\Phi=I_{s} \Lambda+M I_{L_{t}}$ and $\varphi=I_{L_{t}} L_{t}+$ $M I_{s}$. Current conservation in the two electrically isolated parts of this coupled system implies that $I_{C_{t}}+I_{R_{t}}+$ $I_{L_{t}}=I_{\text {in }}(t)$ and $I_{C}+I_{R}+I_{J}+I_{\Lambda}=0$, where $I_{J}=$ $I_{\text {crit }} \sin \left(2 \pi \Phi / \Phi_{0}\right)$ is the weak link Josephson current, the subscripts $C_{t}, L_{t}, R_{t}, C, \Lambda$, and $R$ refer, respectively, to the currents flowing in the various elements of the tank circuit and SQUID ring, and $I_{\text {in }}(t)$ may include both coherent and noise terms [4,8]. Expressing the circuit current elements above in terms of magnetic fluxes leads to the coupled equations

$$
\begin{aligned}
C_{t} \frac{d^{2} \varphi}{d t^{2}}+\frac{1}{R_{t}} \frac{d \varphi}{d t}+\frac{\varphi}{L_{t}\left(1-K^{2}\right)}= & I_{\mathrm{in}}(t) \\
& +\frac{\mu \Phi}{\Lambda\left(1-K^{2}\right)} \\
C \frac{d^{2} \Phi}{d t^{2}}+\frac{1}{R} \frac{d \Phi}{d t}+I_{\text {crit } \sin \left(\frac{2 \pi \Phi}{\Phi_{0}}\right)} & + \\
\frac{\Phi}{\Lambda\left(1-K^{2}\right)} & =\frac{\mu \varphi}{\Lambda\left(1-K^{2}\right)},
\end{aligned}
$$

where $\mu\left(=M / L_{t}\right)$ is the fraction of rf flux $\varphi$ coupled between the tank circuit and the ring. In general, a bias flux $\Phi_{\text {xqstat }}$ is also applied. For convenience we assume that the current generating this is fed through the rf coil (i.e., $\mu$ is the same for both the fluxes seen by the ring).

In the RSJ $+C$ model we can write down characteristic times $\tau_{\mathrm{sqcr}}=C R$ and $\tau_{\mathrm{sq} \lambda \mathrm{c}}=2 \pi \sqrt{\Lambda C}$ for the ring and $\tau_{t}=1 / f_{R}$ for the tank circuit. For parameter values typically invoked in this model (e.g., $C \approx 10^{-12}$ to $10^{-13} F, \Lambda \simeq$ a few $\times 10^{-10} H$, and $R \approx 1$ to $10 \Omega$ ), and $f_{R} \approx 20 \mathrm{MHz}, \tau_{\mathrm{sqcr}}$ and $\tau_{\mathrm{sq} \lambda \mathrm{c}}$ can be 3 or more orders of magnitude smaller than $\tau_{t}$. This means that (1) and (2) must be handled carefully if accurate dynamical solutions for the system are to be found. For this reason we have made no simplifying assumptions. In particular, we do not neglect the derivative terms in (2). We solve the coupled equations using fourth order Runge-Kutta with an adaptive step size algorithm and a sufficient number of steps per rf cycle to follow the oscillations in the SQUID ring accurately. Typically, we find that we can obtain reliable solutions when our integrations are taken over a few thousand steps per rf cycle. 
To make comparison with experiment we need to compute $V_{\text {out }}$ vs $I_{\text {in }}$ at a particular value of rf frequency. It is common experimental practice in the hysteretic regime to adjust the rf so that $\left|V_{\text {out }}\right|$ vs $I_{\text {in }}$. For relatively large risers (where $\beta \gg 1$ ) this means driving at, or very close to, the system resonant frequency at which the ring is most diamagnetic, i.e., at the $\Phi_{\mathrm{xqstat}}=n \Phi_{0}$ bias frequency $f_{\mathrm{rf}}$ where $I_{s}$ is almost linear in $\Phi_{x}$. We refer to this as the pseudoresonant frequency $[4,8]$ given by $f_{R}\left\{1-\left[K^{2} \chi\left(n \Phi_{0}\right) /\left(1-K^{2}\right)\right]\right\}^{1 / 2}$, with a ring magnetic susceptibility $\chi\left(n \Phi_{0}\right)=\partial\left\langle I_{s}\left(n \Phi_{0}\right)\right\rangle / \partial \Phi_{x}=-1$.

As we have shown [10], we can estimate $C$ if we know $I_{\text {crit }}$ and the Fermi velocity of the ring material (niobium). Thus, for $I_{\text {crit }} \cong 50 \mu A, C_{\text {estimated }}$ is close to $10^{-13} \mathrm{~F}$, a value considered typical for high critical current point contact SQUID rings. Within the RSJ $+C$ model the range of $R$ can be arrived at by measuring the dc voltagecurrent characteristics of singly connected point contact weak links, e.g., for $I_{\text {crit }} \cong 50 \mu \mathrm{A}, R$ is typically $\sim 10 \Omega$. Accordingly, for a best fit to the data of Fig. 1, Eqs. (1) and (2) were solved for $I_{\text {crit }}=43 \mu \mathrm{A}, C=10^{-13} \mathrm{~F}$, with $R$ taken as an adjustable parameter between 5 and $30 \Omega$. The other ring-tank circuit parameters were set as for the
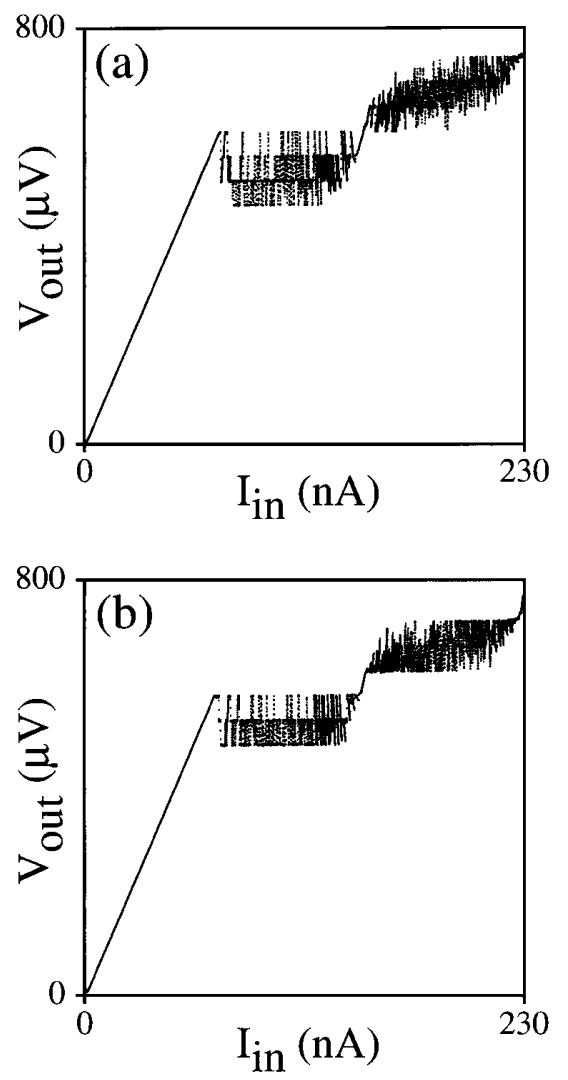

FIG. 4. Computed $V_{\text {out }}$ vs $I_{\text {in }}\left(C=10^{-13} \mathrm{~F}, R=19 \Omega+\right.$ $4 \mathrm{~K}$ noise) at (a) $\Phi_{\text {xqstat }}=n \Phi_{0}$ and (b) $(n+1 / 2) \Phi_{0}$ for the circuit parameters of Fig. 1. experimental dynamics of Fig. 1. For accuracy a simulated $4 \mathrm{~K}$ noise source $[4,8]$, derived from a random number generator, was included in $I_{\text {in }}$. The computed best fit extremal characteristics (for $R=19 \Omega$ ) are shown in Fig. 4. Here, it can be seen that (i) for these chosen values of $C$ and $R$ at least, the RSJ $+C$ model predicts the existence of sublevels, as indicated by earlier computations [7], (ii) the number of sublevels in each gross structure changes with $\Phi_{\text {xqstat }}$ in the manner observed experimentally, with a good match to the data of Fig. 1, and (iii) stochastic jumps are generated between the sublevels which follow closely those observed experimentally. Extensive computations have demonstrated that sublevels are generated only when $I_{\text {crit }}$ is large $(\beta \gg 1)$. Its value, in turn, dictates the range of $C$ and $R$. We note that by computation the maximum number of levels generated so far is 15 , strictly comparable with the maximum of 13 detected experimentally.

In this paper we have demonstrated the existence of multiple levels in the dynamical response of an experimental $\mathrm{rf}$ SQUID magnetometer in the strongly hysteretic regime. These levels, $\Phi_{0}$ periodic in $\Phi_{x}$, are in very good agreement with the theoretical predictions of the full nonlinear RSJ $+C$ model for the coupled system. Our results represent a significant improvement in the experimental sensitivity and, together with the predictions of the model, clearly expose a new and potentially very rich field of nonlinear behavior in an extremely interesting system.

*Email address: t.d.clark@ sussex.ac.uk

[1] K. K. Likharev, Dynamics of Josephson Junctions and Circuits (Gordon and Breach, Sidney, 1986).

[2] W. C. Schieve, A. R. Bulsara, and E.W. Jacobs, Phys. Rev. A 37, 3541 (1988).

[3] M.P. Soerensen, M. Barchelli, P.L. Christiansen, and A. R. Bishop, Phys. Lett. 109A, 347 (1985).

[4] T.D. Clark, J.F. Ralph, R. J. Prance, H. Prance, J. Diggins, and R. Whiteman, Phys. Rev. E 57, 4035 (1998).

[5] L. Gammaitoni, P. Hänggi, P. Jung, and F. Marchesoni, Rev. Mod. Phys. 70, 1 (1998).

[6] O. V. Lounasmaa, Experimental Principles and Methods below 1 K (Academic Press, London, 1974), pp. 156-159.

[7] E. Ben-Jacob and D. Abraham, Appl. Phys. Lett. 39, 835 (1981).

[8] R. Whiteman, J. Diggins, V. Schöllmann, T. D. Clark, R. J. Prance, H. Prance, and J. F. Ralph, Phys. Lett. A 234, 205 (1997).

[9] J. E. Zimmerman, P. Thiene, and J. T. Harding, J. Appl. Phys. 41, 1572 (1970).

[10] J.F. Ralph, T.D. Clark, J. Diggins, R. J. Prance, H. Prance, and A. Widom, J. Phys. Condens. Matter 9, 8275 (1997). 